\title{
Hypoxia-induced Maspin Expression Affects the Prognosis of Ovarian Clear Cell Carcinoma
}

\author{
EUN JI LEE ${ }^{1}$, SOO JIN PARK ${ }^{1}$, CHEOL LEE ${ }^{2}$, GA WON YIM ${ }^{3}$, JAE WEON KIM ${ }^{1}$ and HEE SEUNG KIM ${ }^{1}$ \\ ${ }^{1}$ Department of Obstetrics and Gynecology, \\ Seoul National University College of Medicine, Seoul, Republic of Korea; \\ ${ }^{2}$ Department of Pathology, Seoul National University College of Medicine, Seoul, Republic of Korea; \\ ${ }^{3}$ Department of Obstetrics and Gynecology, Dongguk University College of Medicine, Goyang, Republic of Korea
}

\begin{abstract}
Background/Aim: To investigate the role of the expression of hypoxia-related genes on the prognosis of ovarian clear cell carcinoma (OCCC). Materials and Methods: Basal mRNA levels of eight hypoxia-related genes were compared. Cell viability was assayed after treating ES2 cells under hypoxic conditions. The $m R N A$ and protein levels were evaluated after the induction of hypoxia and administration of increased doses of $N$-acetylcysteine (NAC). Finally, the prognostic role of their expression levels was evaluated in 61 patients with OCCC. Results: The mRNA and protein levels of maspin increased gradually with the induction of hypoxia. Maspin protein expression decreased after treatment with paclitaxel and NAC. High expression of maspin was related to poor progression-free and overall survival in patients with OCCC (adjusted hazard ratios, 3.97 and 7.47; 95\% confidence intervals $=1.34-11.81$, and 1.9828.13). Conclusion: High expression of maspin induced by hypoxia might be associated with poor prognosis of OCCC.

Ovarian cancer is a heterogeneous group of diseases and consists of different histologic types, demonstrating distinct biological properties (1). Even though type II ovarian cancers, including high grade serous carcinoma, have valuable biomarkers such as CA-125 and human epididymis protein 4 , there are few diagnostic or prognostic biomarkers for type I ovarian cancers representing low-grade serous, mucinous, endometrioid, and clear cell carcinoma (2). Thus,
\end{abstract}

This article is freely accessible online.

Correspondence to: Hee Seung Kim, MD, Ph.D., Department of Obstetrics and Gynecology, Seoul National University College of Medicine, 101 Daehak-Ro Jongno-Gu, Seoul 03080, Republic of Korea. Tel: +82 220724863, Fax: + 82 27623599, e-mail: bboddi0311@gmail.com

Key Words: Clear cell, hypoxia, maspin, ovarian cancer, prognosis. it is difficult to predict the clinical prognosis of type I ovarian cancers due to the lack of biomarkers (3). Since previous studies demonstrated the correlation between hypoxia and ovarian cancer progression $(4,5)$, further investigations for identifying new biomarkers related to hypoxia are still meaningful. Among them, ovarian clear cell carcinoma (OCCC) shows rather unique entity in terms of clinical, genetic, and histological features (6). Notably, chronic inflammation and oxidative stress in precancerous lesions such as endometriosis have been suggested to trigger its malignant transformation to $\operatorname{OCCC}(7,8)$. Although hypoxia-inducible factor-1 $\alpha$ (HIF-1 $\alpha$ ) and vascular endothelial growth factor (VEGF) are related to carcinogenesis and progression of ovarian cancer by inducing chemo-resistance (9-11), other hypoxia-related genes such as HIF-1 $\beta$, cAMP-response element-binding protein-binding protein (CBP), adenovirus early region 1Abinding protein P300 (P300), factor-inhibiting hypoxiainducible factor (FIH), von Hippel-Lindau (VHL), and maspin have not been investigated sufficiently for their roles affecting drug resistance and prognosis. There is also a lack of relevant studies about the role of hypoxia-related genes as prognostic factors in ovarian cancer. Thus, this study aimed to investigate the role of the expression of hypoxia-related genes as potential prognostic biomarkers in type I ovarian cancer, especially OCCC.

\section{Materials and Methods}

Reagents, cell lines, and tissue samples. Cisplatin and $\mathrm{N}$ acetylcysteine (NAC) were acquired from Sigma-Aldrich (St. Louis, MO, USA), and paclitaxel was donated by Samyang Biopharmaceuticals Corp. (Seongnam-si, Republic of Korea). For western blot and immunohistochemistry, anti-HIF-1 $\alpha$ was obtained from Abcam (Cambridge, UK). Anti-CBP, anti-FIH, an-ti-HIF-1 $\beta$, and anti-VHL antibodies were purchased from Cell Signaling Technology (Danvers, MA, USA). Moreover, anti-P300, anti-VEGF, and anti-maspin antibodies were obtained from Santa Cruz Biotechnology (Dallas, TX, USA). 
ES-2, a clear cell carcinoma cell line of human ovarian origin (12), and five frozen tissues, each from either a normal ovary or OCCC, were purchased from the Cancer Tissue Bank of Seoul National University (Seoul, Republic of Korea).

Cell culture. ES-2 cells were cultured in a 1:1 mixture of MCDB 105/Medium 199 (Sigma-Aldrich, St. Louis, MO, USA) supplemented with $15 \%$ fetal bovine serum, $100 \mathrm{U} / \mathrm{ml}$ penicillin, and $100 \mu \mathrm{g} / \mathrm{ml}$ streptomycin. ES-2 cells were grown in the culture media until reaching up to $70 \%$ confluency in monolayers in 100$\mathrm{mm}$ tissue culture dishes; subsequently, cells were serum-starved for $24 \mathrm{~h}$ before the assays.

Incubation under normoxic and hypoxic conditions. For normoxic conditions, ES-2 cells were grown in a standard humidified incubator containing $5 \% \mathrm{CO}_{2}$ and $21 \% \mathrm{O}_{2}$ at $37^{\circ} \mathrm{C}$. For hypoxic conditions, cells were incubated in a commercially available hypoxia incubator chamber (STEMCELL Technologies Inc., Vancouver, Canada) containing $90 \% \mathrm{~N}_{2} ; 5 \% \mathrm{CO}_{2} ; 5 \% \mathrm{O}_{2}, 92 \% \mathrm{~N}_{2}$; $5 \% \mathrm{CO}_{2} ; 3 \% \mathrm{O}_{2}$, and $94 \% \mathrm{~N}_{2} ; 5 \% \mathrm{CO}_{2} ; 1 \% \mathrm{O}_{2}$.

Cytotoxicity and cell viability assays. The cultured ES-2 cells were treated with gradually escalated doses of paclitaxel, and cisplatin from 0 to $100 \mu \mathrm{mol} / 1$ was applied to cultured ES-2 cells for determining the half-maximal inhibitory concentration $\left(\mathrm{IC}_{50}\right)$ under normoxic conditions. The MTT [3-(4,5-dimethylthiazol-2yl)-2,5- diphenyltetrazolium bromide] colorimetric assay was performed for assessing cell viability. Following serum starvation for $24 \mathrm{~h}$, MTT solution at $5 \mathrm{mg} / \mathrm{ml}$ was applied to ES-2 cells for $4 \mathrm{~h}$ at $37^{\circ} \mathrm{C}$. After that, the medium was substituted with $100 \mu \mathrm{l}$ of $0.04 \mathrm{~N} \mathrm{HCl}$ in isopropanol to dissolve the formazan crystals. Cell viability was quantified with a microplate reader (Bio-rad, Seoul, Republic of Korea) at $595 \mathrm{~nm}$ and $655 \mathrm{~nm}$ as a background. The percentage of cell viability was calculated according to the following equation:

Cell viability percentage $(\%)=($ absorbance of hypoxia or chemical compound-treated cells divided by absorbance of control cells) $\times 100$.

Quantitative real time polymerase chain reaction assay. Total cellular RNA of ES-2 cells and each frozen tissue derived from either normal ovary or OCCC were extracted by utilizing Trizol reagent (Invitrogen, Carlsbad, CA, USA) based on the manufacturer's guidelines. Complementary DNA was synthesized using total RNA extracted from ES-2 cells with the AccuPower RT PreMix (Bioneer, Daejeon, Republic of Korea). Gene expression was measured using SYBR Green (Sigma) and a StepOnePlus qRTPCR system (Applied Biosystems, Foster City, CA, USA). For the control and normalization of variation, the glyceraldehyde-3phosphate dehydrogenase $(G A P D H)$ gene expression was analyzed simultaneously in each experiment. The sequences of the forward and reverse primers of the eight hypoxia-related genes are shown in Supplementary Table I. The forward primer (5'-ACA CAG AAG ACG GTG GAT GG-3') and the reverse primer (5'-GGC AGG TCA GGT CAA CAA CA-3') for the GAPDH gene were constructed for the amplification of $G A P D H$, a 193 bp product. Subsequently, expression levels of the eight hypoxia-related genes were determined using the cycle threshold (CT) values and the standard curve method. The values were normalized based on the expression of the GAPDH gene.
The conditions of polymerase chain reaction (PCR) were the following: denaturation at $95^{\circ} \mathrm{C}$ for $3 \mathrm{~min}$, followed by 40 cycles at $95^{\circ} \mathrm{C}$ for $30 \mathrm{~s}$, annealing at $60^{\circ} \mathrm{C}$ for $30 \mathrm{~s}$, and DNA synthesis at $72^{\circ} \mathrm{C}$ for $30 \mathrm{~s}$. PCR was performed using a melting curve program, which increased the temperature at a rate of $0.5^{\circ} \mathrm{C}$ every $10 \mathrm{~s}$, ranging from $55^{\circ} \mathrm{C}$ to $95^{\circ} \mathrm{C}$ with a continuous fluorescence measurement. ROX reference dye (Invitrogen, Carlsbad, CA, USA) was used as a negative control for the fluorescence measurements. Sequence-specific products were identified by generating a melting curve, where $\mathrm{CT}$ value represented the cycle number at which a fluorescent signal was statistically more significant than the background. Relative gene expression levels were quantified using the 2- $\Delta \Delta$ CTmethod (13)

Determination of the levels of intracellular reactive oxygen species. Intracellular reactive oxygen species (ROS) production was evaluated by using 2',7'-dichlorofluorescein diacetate (DCFH-DA, Sigma), which was converted to fluorescent 2',7'dichlorofluorescein (DCF) in the presence of peroxides. ES-2 cells were detached with trypsin-ethylenediaminetetraacetic acid (EDTA), collected by centrifugation, and washed with phosphate-buffered saline (PBS). The cells were treated with $10 \mu \mathrm{M}$ DCFH-DA for 30 min at $37^{\circ} \mathrm{C}$. Then, the cells were washed with PBS twice and treated with NAC in a dose-dependent manner $(0,1,2,5$, and 10 $\mathrm{mM}$ ) for $1 \mathrm{~h}$ at $37^{\circ} \mathrm{C}$ in a $\mathrm{CO}_{2}$ incubator, with a combination of either paclitaxel or cisplatin. The treated cells were re-washed with PBS. Fluorescent DCF intensity was analyzed using a flow cytometer (BD Bioscience, Franklin Lakes, NJ, USA).

Western blot. Protein concentration of CBP, P300, HIF-1 $\alpha$, HIF$1 \beta$, FIH, VHL, VEGF, and maspin in whole-cell extracts was estimated using the Bradford protein assay (Bio-Rad, Hercules, CA, USA) with bovine serum albumin as the standard. Protein extract was denatured, separated using sodium dodecyl sulphatepolyacrylamide gel electrophoresis (SDS-PAGE), transferred to a nitrocellulose membrane and developed through enhanced chemiluminescence detection (Super-Signal, West Pico, IL, USA). The protein amount was quantified by measuring light intensity from correctly sized bands under ultraviolet light using a ChemiDoc EQ system and Quantity One software (Bio-Rad). Detection of immunore-active proteins was done using goat antirabbit polyclonal antibodies against phospho-proteins and totalproteins at a 1:1,000 dilution and 10\% SDS/PAGE gel. Total protein levels were used as a loading control for the normalization of results from protein detection by western blotting. Multiple exposures of each western blot were performed to ensure the linearity of chemiluminescent signals.

Human population. The clinicopathological data of 61 patients with OCCC between July 2001 and November 2012 were gathered. The inclusion criteria were as follows: diagnosis of OCCC; primary cytoreductive surgery followed by adjuvant chemotherapy using paclitaxel $\left(175 \mathrm{mg} / \mathrm{m}^{2}\right)$ and cisplatin $(50$ $\left.\mathrm{mg} / \mathrm{m}^{2}\right)$; Eastern Cooperative Oncology Group (ECOG) performance status ranging from $0-2$; and no underlying diseases which may affect survival.

Data on the patients' stage based on the International Federation of Gynecology and Obstetrics (FIGO) classification, age, the extent of cytoreductive surgery, platinum-resistance, progression-free survival (PFS), and overall survival (OS) were extracted. Optimal 
cytoreduction was defined as cytoreductive surgery with a maximal diameter of residual tumors measured $\leq 1 \mathrm{~cm}$. Platinum resistance was defined as a treatment-free interval less than six months following completion of adjuvant chemotherapy. PFS was defined as the period between the date of surgery and the date of disease recurrence, and OS was defined as the period from the date of surgery to the date of death or end of this study.

Immunohistochemistry. We conducted immunohistochemistry (IHC) to evaluate the expression of the eight hypoxia-related genes in patients with OCCC. For the negative control, purified non-immune mouse $\operatorname{IgG}$ as primary antibody substitution was utilized at the same concentration. Representative sections of core tissue $(2 \mathrm{~mm}$ diameter) were sliced from paraffin blocks and arranged in new tissue microarray blocks using the trephine apparatus (SuperBioChips Laboratories, Seoul, Republic of Korea). Subsequently, a pathologist who was unaware of the patients' clinicopathological characteristics interpreted and scored the eight hypoxia-related genes' expression levels semi-quantitatively. The scoring system was based on two parameters - the product of staining intensity, read as I score (1, weak; 2, moderate; and 3, strong) and the percentage of positive cells, read as P score (1, $<25 \% ; 2,25-50 \% ; 3,51-75 \%$; and $4,>75 \%$ ). The immunoreactivity score was defined as I score multiplied by P score, ranging from 1 to 12 . Protein expression was considered high when the score was six or more because the median score was six.

Statistical analysis. The study was conducted according to the guidelines of the Declaration of Helsinki, and approved by the Institutional Review Board of Seoul National University Hospital (No. 1311-050-533). Informed consent was waived due to the retrospective design of the study and there were no ethical risks.

All in vitro experiments were replicated three times for obtaining consistent results, and cell viability assays were analyzed by the analysis of variance (ANOVA) method based on the general linear model (PROC-GLM) of the SAS program (SAS Institute Inc., Cary, NC, USA). For identifying the roles of the eight hypoxia-related genes in influencing platinum resistance and PFS, the Chi-square test and Student's $t$-test were performed. KaplanMeier analysis with the log-rank test, Cox's proportional hazard, and logistic regression analyses were done to determine the hazard ratio (HR), odds ratio (OR), and $95 \%$ confidence interval (CI). Statistical analysis was performed using SPSS software version 21.0 (SPSS Inc., Chicago, IL, USA). A $p$-value $<0.05$ was considered statistically significant.

\section{Results}

Cell viability. $\mathrm{IC}_{50}$ values of the chemotherapeutic agents paclitaxel and cisplatin were 20 and $50 \mu \mathrm{M}$, respectively (Supplementary Figure 1). After determining $\mathrm{IC}_{50}$ values, ES-2 cells were treated with paclitaxel $(20 \mu \mathrm{M})$ and cisplatin $(50 \mu \mathrm{M})$ under different hypoxic conditions. Accordingly, ES-2 cell proliferation was less inhibited under normoxic conditions, and an increase in hypoxia had a minor influence on the inhibition of cell growth (Supplementary Figure 2). These results suggest that hypoxia might be associated with chemo-resistance of OCCC cells.
mRNA expression. We compared the mRNA levels of CBP, P300, HIF-1 $\alpha$, HIF-1 $\beta$, FIH, VHL, VEGF, and maspin between normal ovarian and OCCC tissues under normoxic conditions. The mRNA levels of VEGF were similar between normal ovarian and OCCC tissues. In contrast, the mRNA levels of CBP, P300, HIF-1 $\alpha$, HIF-1 $\beta$, FIH, VHL, and maspin were lower in OCCC than in normal ovarian tissues (Supplementary Figure 3). Next, we compared changes in the eight hypoxia-related genes' mRNA levels after treating ES2 cells with paclitaxel and cisplatin under hypoxic conditions. There were no changes in the mRNA levels of CBP, HIF-1 $\alpha$, and VEGF, whereas the mRNA levels of HIF$1 \beta$, FIH, and VHL showed inconsistent changes after administration of paclitaxel or cisplatin under hypoxia. Moreover, only the mRNA levels of maspin increased gradually after administration of paclitaxel or cisplatin under hypoxic conditions (Figure 1).

Effects of $N$-acetylcysteine on cell proliferation and reactive oxygen species production. To evaluate the relationship between hypoxia and ROS generation in ES-2 cells, we estimated cellular proliferation and ROS production in ES-2 cells after treatment with NAC, a potent anti-oxidant. Cell proliferation was not affected after treatment with 0 to 10 $\mathrm{mM}$ of NAC, whereas it was decreased after treatment with $20 \mathrm{mM}$ of NAC. Moreover, ROS production was gradually reduced after treatment with NAC. Then, we evaluated ROS production in ES-2 cells treated with either paclitaxel or cisplatin in the presence of increased concentrations of NAC. The addition of 0 to $5 \mathrm{mM}$ of NAC after treatment with paclitaxel resulted in a gradual decrease in ROS production. However, there was no change in ROS production after administration of cisplatin in ES-2 cells despite the presence of NAC (Figure 2).

Protein expression. Administration of paclitaxel or cisplatin under hypoxic conditions had no effect on the protein expression of CBP, P300, HIF-1 $\alpha$, HIF-1 $\beta$, FIH, and VEGF. However, VHL protein expression of decreased after administration of cisplatin, and that of maspin increased after administration of paclitaxel under hypoxic conditions. On the other hand, maspin protein expression decreased after administration of paclitaxel in the presence of increased concentrations of NAC in ES-2 cells (Figure 3).

Prognosis. The clinicopathological characteristics of 61 patients diagnosed with OCCC are summarized in Table I. The median age was 49 years, and 40 patients $(65.6 \%)$ had early-stage OCCC (stage I-II disease). Moreover, 53 (86.9\%) and 47 patients $(77 \%)$ received optimal cytoreduction and six or more cycles of chemotherapy. Nuclear expression of CBP, HIF-1 $\beta$, VHL, maspin, and cytoplasmic expression of P300, HIF-1 $\alpha$, FIH were observed by IHC (Supplementary 

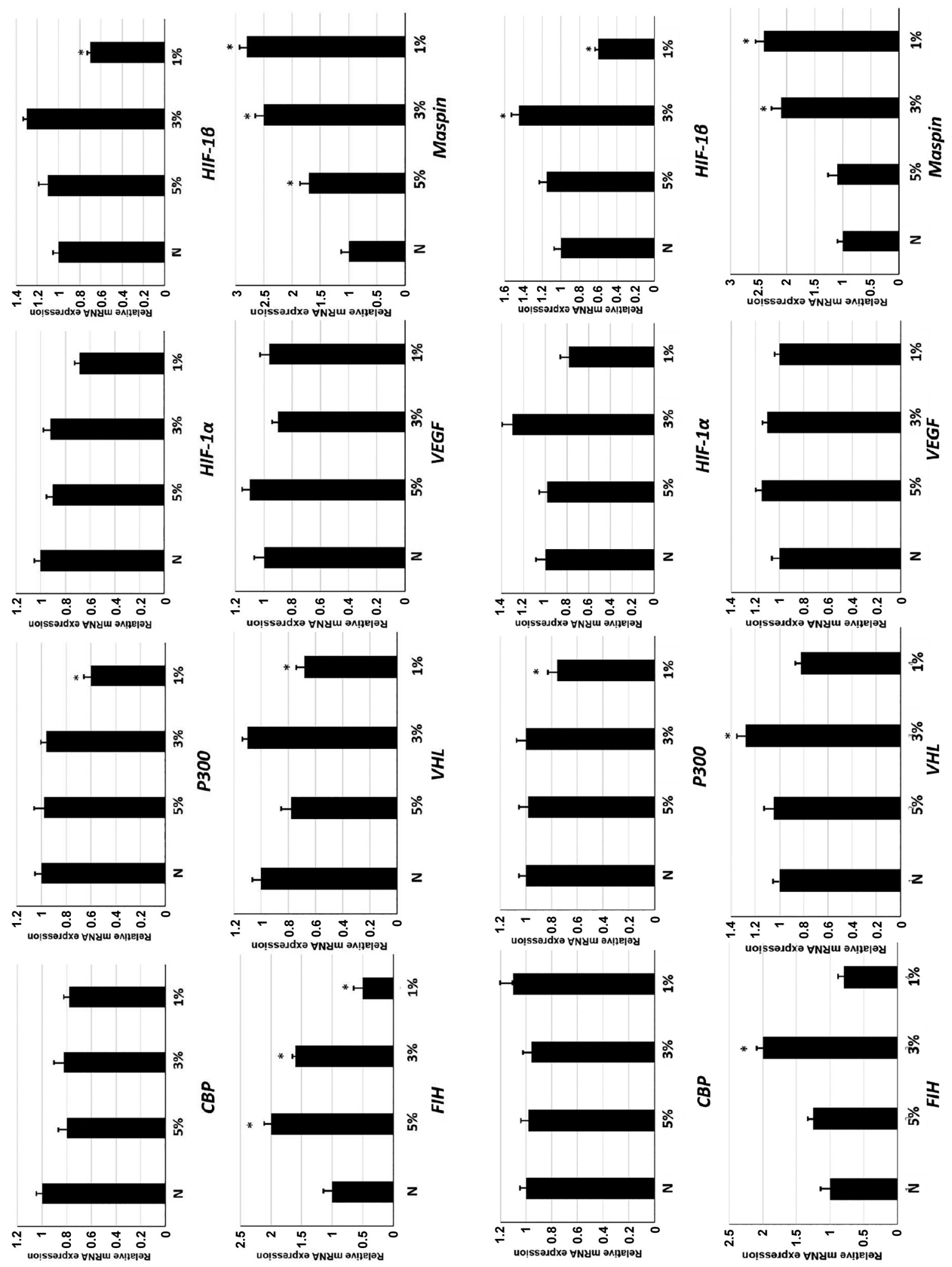

$\varangle$

$m$ 
A

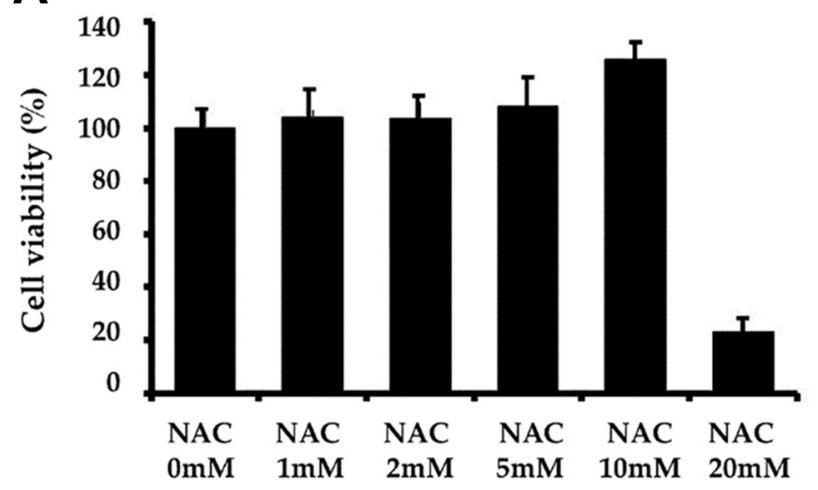

C

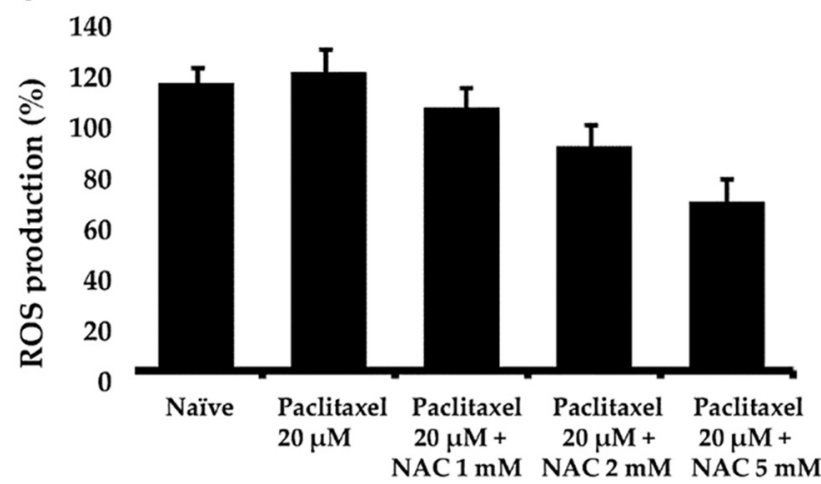

B

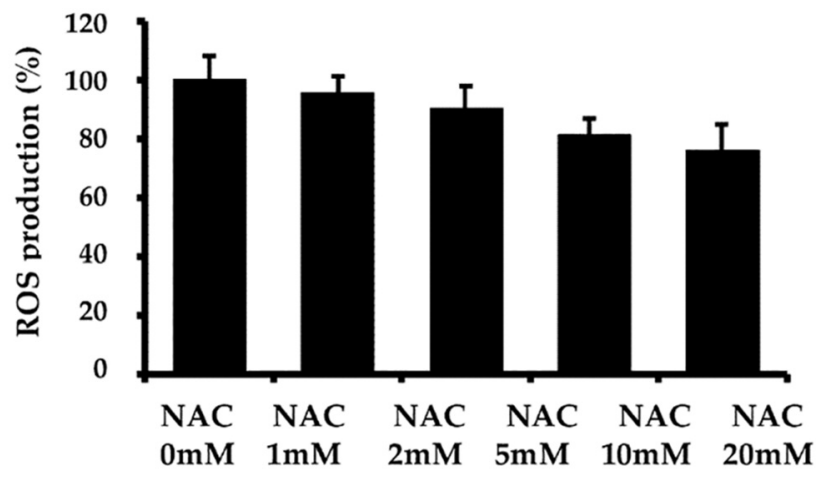

D

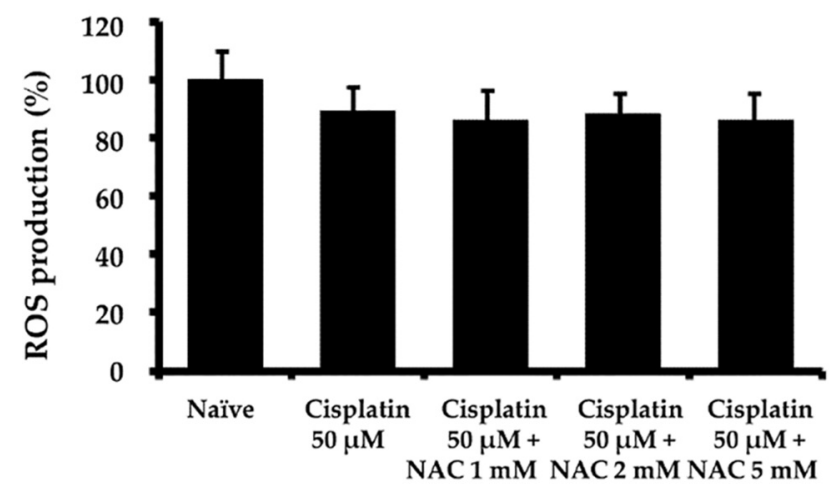

Figure 2. Cell viability and comparison of reactive oxygen species (ROS) production. (A) Cell proliferation following treatment with $N$-acetylcysteine $(N A C)$; ROS production $(B)$ after treatment with NAC, $(C)$ after treatment with paclitaxel, and $(D)$ after treatment with cisplatin in the presence of increased concentrations of NAC.

A

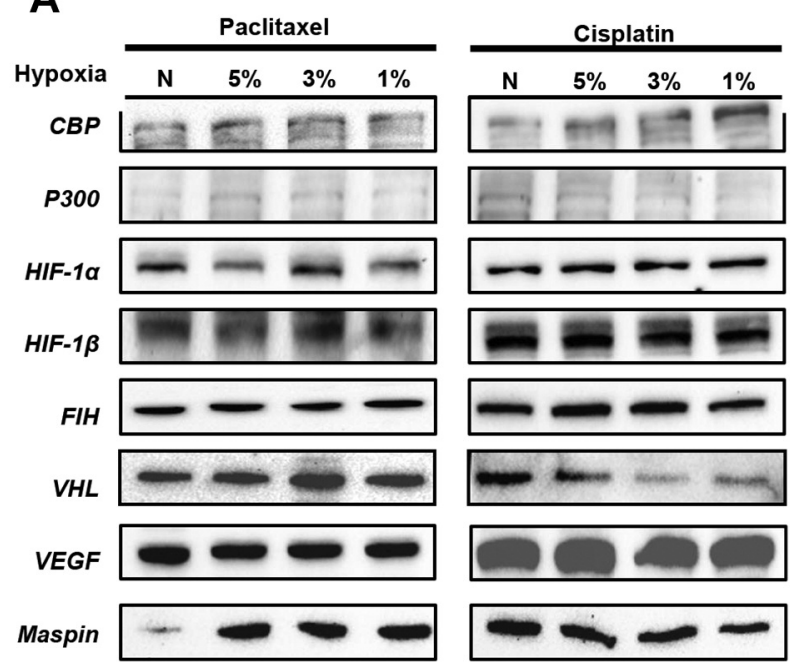

B

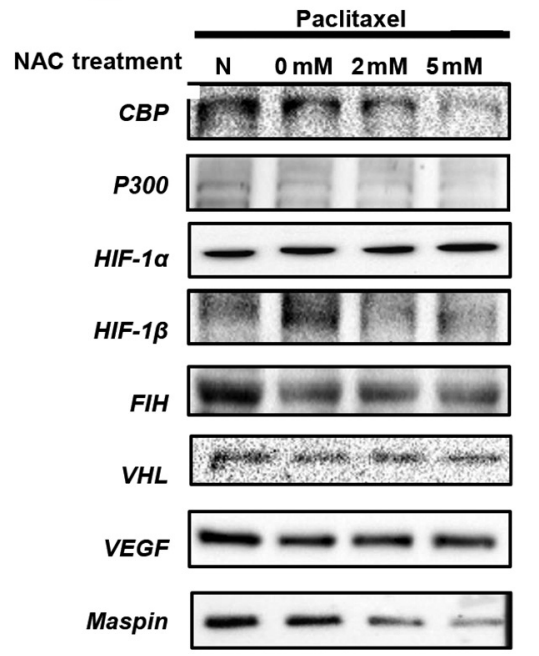

Figure 3. Comparison of the expression of cAMP-response element-binding protein-binding protein (CBP), adenovirus early region $1 A$-binding

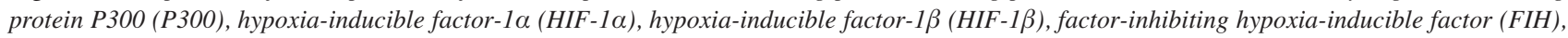
von Hippel-Lindau (VHL), vascular endothelial growth factor (VEGF) and maspin proteins after treatment with paclitaxel and cisplatin (A) under hypoxic conditions and $(B)$ in the presence of increasing concentrations of $N$-acetylcysteine (NAC), in ES-2 cells by western blot. 
A

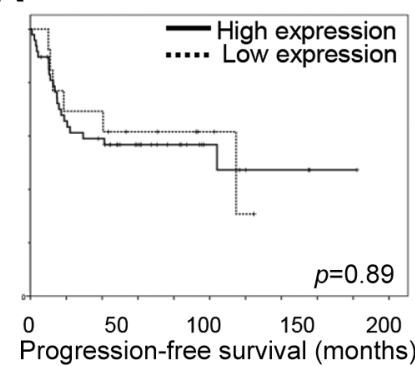

CBP

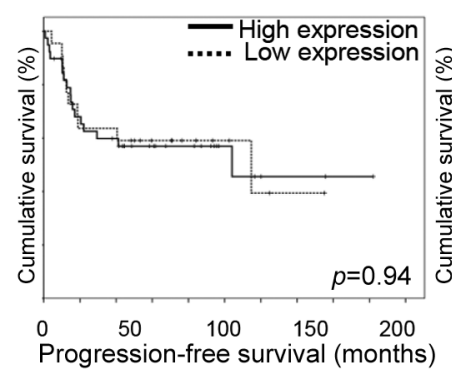

FIH

B
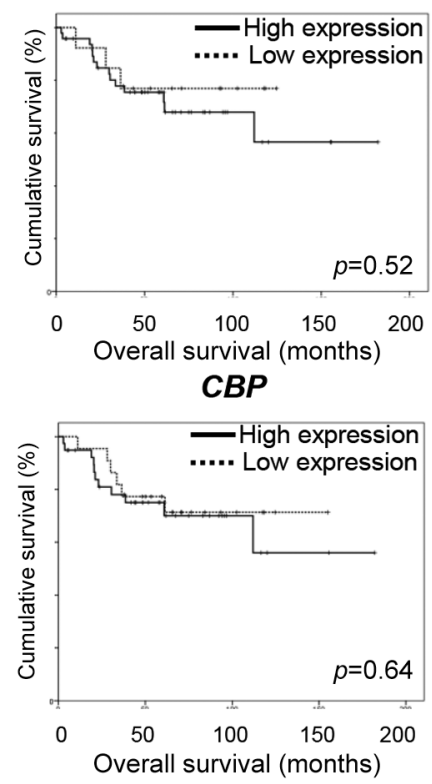

FIH

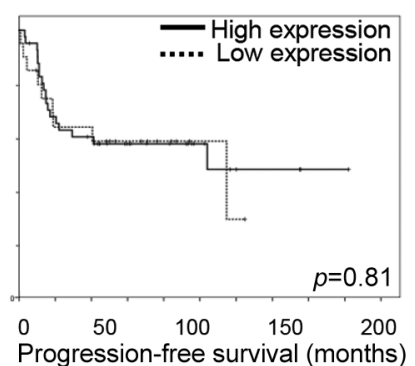

P300

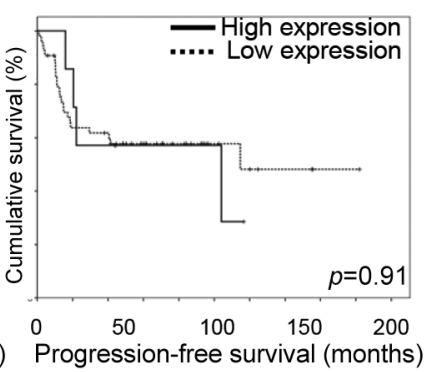

VHL

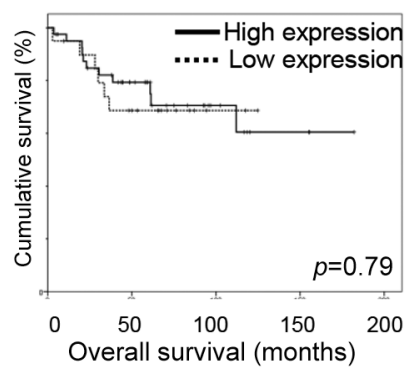

$P 300$

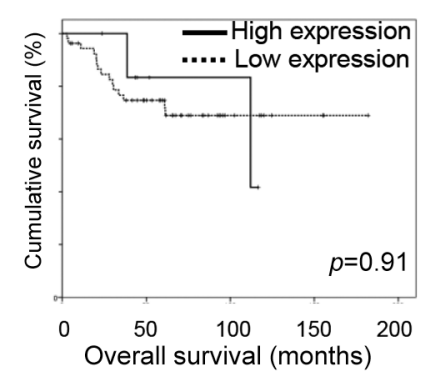

VHL

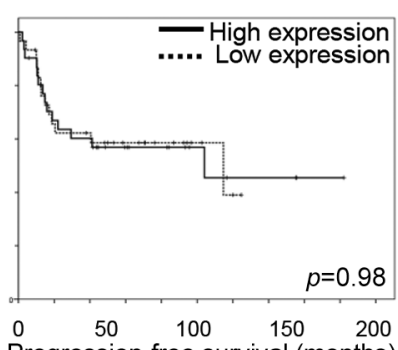

Progression-free survival (months)

HIF-1a

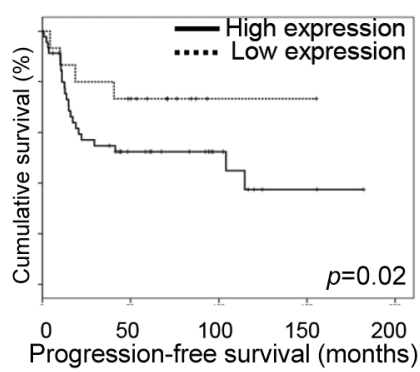

maspin

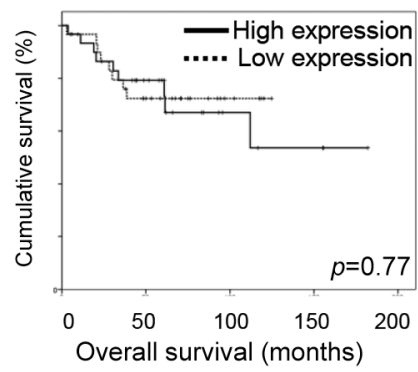

HIF-1a

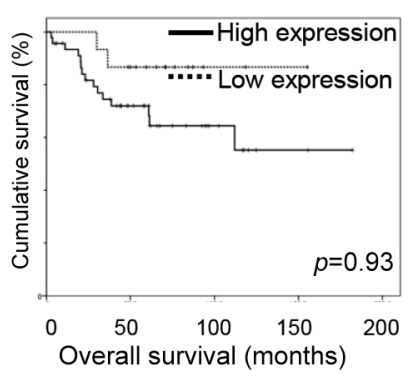

maspin

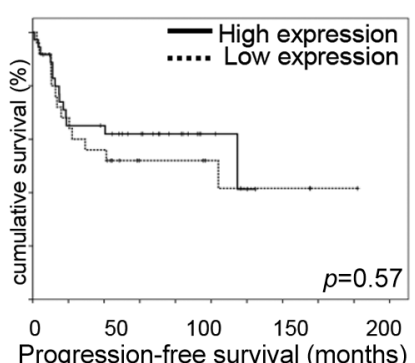

HIF-1及

Figure 4. Comparison of (A) progression-free survival and $(B)$ overall survival between low and high expression of the eight hypoxia-related genes including cAMP-response element-binding protein-binding protein (CBP), adenovirus early region 1 A-binding protein P300 (P300), hypoxiainducible factor-1 $\alpha$ (HIF-1 $\alpha)$, hypoxia-inducible factor-1 $\beta$ (HIF-1 $\beta)$, factor-inhibiting hypoxia-inducible factor (FIH), von Hippel-Lindau (VHL) and maspin in patients with ovarian clear cell carcinoma.

Figure 4). Supplementary Table II depicts factors affecting platinum resistance in univariate and multivariate analyses. Only FIGO stage III-IV disease and suboptimal cytoreduction were risk factors for platinum resistance (adjusted ORs, 27.2 and 12.9; 95\% CI=2.54-29.59, and 1.839.04; $p<0.05$ ).

In terms of survival, high expression of maspin was related to worse PFS and OS compared to low expression of 
Table I. Characteristics of 61 patients with clear cell carcinoma of the ovary.

\begin{tabular}{llc}
\hline Characteristics & & $\mathrm{N}=61(\%)$ \\
\hline Age (years) & $<49$ & $30(49.2)$ \\
& $\geq 49$ & $31(50.8)$ \\
FIGO stage & I & $36(59)$ \\
& II & $4(6.6)$ \\
& III & $19(31.1)$ \\
Residual tumor (cm) & IV & $2(3.3)$ \\
Cycles of chemotherapy & $\leq 1$ & $53(86.9)$ \\
& $>1$ & $8(13.1)$ \\
CBP expression & $<6$ & $14(23)$ \\
& $\geq 6$ & $47(77)$ \\
P300 expression & High & $48(78.7)$ \\
HIF-1 $\alpha$ expression & Low & $13(21.3)$ \\
& High & $41(67.2)$ \\
HIF-1 $\beta$ expression & Low & $20(32.8)$ \\
& High & $31(50.8)$ \\
FIH expression & Low & $30(49.2)$ \\
& High & $25(41)$ \\
VHL expression & Low & $36(59)$ \\
& High & $39(63.9)$ \\
VEGF expression & Low & $22(36.1)$ \\
& High & $7(11.5)$ \\
Maspin expression & Low & $54(88.5)$ \\
& High & $61(100)$ \\
& Low & $0(0)$ \\
& High & $46(75.4)$ \\
& Low & $15(24.6)$ \\
\hline
\end{tabular}

FIGO: International Federation of Gynecology and Obstetrics; CBP: cAMP-response element-binding protein-binding protein; P300: adenovirus early region 1A-binding protein P300; HIF-1 $\alpha$ : hypoxia inducible factor- $1 \alpha$; HIF-1 $\beta$ : hypoxia inducible factor- $1 \beta$; FIH: factorinhibiting hypoxia inducible factor; VHL: von Hippel-Lindau; VEGF: vascular endothelial growth factor.

maspin (median, PFS, 91.3 vs. 118.7 months; OS, $121.4 v s$. 138.9 months; $p<0.05$; Figure 4 ). In multivariate analyses, high expression of maspin was a prognostic factor for poor PFS and OS (adjusted HRs, 3.97 and 7.47; 95\%CI=1.3411.81 and 1.98-28.13; Table II).

\section{Discussion}

OCCC has been suggested to develop by malignant transformation of precancerous lesions such as endometriosis under chronic inflammation and oxidative stress, unlike other histologic types that may develop by müllerian differentiation or exposure to unopposed estrogen (7). Thus, prognostic values of hypoxia-related genes can differ among various types of histology under hypoxic conditions that exacerbate chronic inflammation and oxidative stress.

This study showed that most hypoxia-related genes such as CBP, P300, HIF-1 $\alpha$, HIF-1 $\beta$, FIH, and VEGF were not overexpressed under hypoxic conditions, which means that their roles in predicting the prognosis of OCCC may be limited. These findings are supported by a previous study that showed glutathione peroxidase 3 (GPX3) over-expression in OCCC compared with other histologic types of ovarian cancer (14). Since the major biochemical function of GPX3 is to catalyze the conversion of glutathione and hydrogen peroxide into glutathione disulfide and water as one of the most important cellular detoxifying mechanisms against oxidative damages (15), GPX3 over-expression in OCCC can suppress hypoxiarelated gene expression, affecting prognosis.

Maspin, a protein encoded by the SERPIN5 gene in chromosome 18q21.3-q23, is classified under the mammalian serine protease inhibitor family and functions as a tumor suppressor gene (16). Although maspin has been suggested to play a role as an anti-angiogenic inhibitor in various types of malignancies (17), its role is still controversial in ovarian cancer because the relatively few reports have demonstrated conflicting results about the relationship between maspin expression and its prognosis (18-21).

This study demonstrated that maspin expression increased after administration of paclitaxel under hypoxic conditions or in the presence of increased concentrations of NAC. These findings are supported by a previous study, which reveal that maspin expression was high in cisplatin-sensitive CAOV-3 and OVCAR-3 cells and low in cisplatin-resistant ES- 2 cells (20). Moreover, high expression of stathmin, a microtubuledestabilizing protein, and maspin have been reported to play a role in the progression of adenoid cystic carcinoma (22), and thereby high expression of stathmin can decrease microtubule stabilization, which might reduce the effect of paclitaxel, in particular, in cancers with wild-type p53 such as OCCC (23).

Moreover, high expression of maspin was associated with poor prognosis of OCCC despite the no relation to platinum resistance. These results suggest that high expression of maspin induced by hypoxia could lead to resistance to paclitaxel and a subsequent worse survival in patients with OCCC. This hypothesis is supported by a previous study where the reduction in the levels of heat shock protein 27 increased chemosensitivity to paclitaxel by increasing ROS production in ovarian cancer (24).

The current study has several limitations. First, clinical outcomes, such as platinum resistance and survival, were reviewed retrospectively. Second, we used only the ES-2 cell line as an OCCC cell line. Considering that there are considerable variations regarding mutation and activity of signaling pathways, the in vitro experiments should be extended to include additional OCCC cell lines. Third, the absence of comparison to normal ovarian cells and investigation of cell viability without chemotherapy under hypoxia as a control limit the interpretation of our results. Fourth, the detailed mechanisms of hypoxia-induced expression of maspin and its relation to the resistance to 
Table II. Factors affecting survival in 61 patients with ovarian clear cell carcinoma.

\begin{tabular}{|c|c|c|c|c|c|c|}
\hline \multirow[b]{2}{*}{ Characteristics } & \multicolumn{3}{|c|}{ Univariate } & \multicolumn{3}{|c|}{ Multivariate } \\
\hline & HR & $95 \% \mathrm{CI}$ & $p$-Value & Adjusted HR & $95 \% \mathrm{CI}$ & $p$-Value \\
\hline \multicolumn{7}{|l|}{ Progression-free survival } \\
\hline Age $<49$ years & 1.26 & $0.59-2.7$ & 0.79 & - & - & - \\
\hline FIGO stage III-IV & 15.3 & $5.81-40.28$ & $<0.01$ & 16.53 & $4.98-54.91$ & $<0.01$ \\
\hline Suboptimal cytoreduction & 8.13 & $3.38-19.61$ & $<0.01$ & 5.47 & $1.79-16.72$ & $<0.01$ \\
\hline$<6$ cycles of chemotherapy & 1.77 & $0.7-4.45$ & 0.23 & - & - & - \\
\hline High expression of CBP & 1.07 & $0.43-2.64$ & 0.89 & - & - & - \\
\hline High expression of $\mathrm{P} 300$ & 0.91 & $0.41-2.02$ & 0.81 & - & - & - \\
\hline High expression of HIF- $1 \alpha$ & 1.01 & $0.48-2.15$ & 0.98 & - & - & - \\
\hline High expression of HIF- $1 \beta$ & 1.25 & $0.58-2.66$ & 0.57 & - & - & - \\
\hline High expression of FIH & 1.03 & $0.47-2.26$ & 0.94 & - & - & - \\
\hline High expression of VHL & 1.07 & $0.37-3.09$ & 0.91 & - & - & - \\
\hline High expression of maspin & 2.74 & $1.23-8.42$ & 0.02 & 3.97 & $1.34-11.81$ & 0.01 \\
\hline \multicolumn{7}{|l|}{ Overall survival } \\
\hline Age $<49$ years & 2.23 & $0.78-6.35$ & 0.13 & - & - & - \\
\hline FIGO stage III-IV & 11.48 & $3.68-35.75$ & $<0.01$ & 52.46 & $8.98-63.63$ & $<0.01$ \\
\hline Suboptimal cytoreduction & 3.64 & $1.16-11.36$ & 0.03 & 20.2 & $2.67-15.25$ & $<0.01$ \\
\hline$<6$ cycles of chemotherapy & 2.77 & $0.95-8.07$ & 0.74 & - & - & - \\
\hline High expression of CBP & 1.50 & $0.43-5.24$ & 0.52 & - & - & - \\
\hline High expression of $\mathrm{P} 300$ & 0.87 & $0.32-2.36$ & 0.79 & - & - & - \\
\hline High expression of HIF- $1 \alpha$ & 1.15 & $0.44-2.99$ & 0.77 & - & - & - \\
\hline High expression of HIF-1 $\beta$ & 1.56 & $0.58-4.05$ & 0.36 & - & - & - \\
\hline High expression of FIH & 1.27 & $0.47-3.44$ & 0.64 & - & - & - \\
\hline High expression of VHL & 0.91 & $0.21-4.02$ & 0.91 & - & - & - \\
\hline High expression of maspin & 3.04 & $1.69-13.31$ & 0.03 & 7.47 & $1.98-28.13$ & $<0.01$ \\
\hline
\end{tabular}

HR: Hazard ratio; CI: confidence interval; FIGO: International Federation of Gynecology and Obstetrics; CBP: cAMP-response element-binding protein-binding protein; P300: adenovirus early region 1A-binding protein P300; HIF-1 $\alpha$ : hypoxia inducible factor-1 $\alpha$; HIF-1 $\beta$ : hypoxia inducible factor-1 $\beta$; FIH: factor-inhibiting hypoxia inducible factor; VHL: von Hippel-Lind.

paclitaxel were not evaluated. Fifth, mRNA levels were inconsistent among some genes despite the repeated experiments, which should be further investigated considering the interaction of these genes in relation to hypoxia and chemotherapy. Sixth, the prognostic value of maspin should be validated in further studies including a large number of patients.

In conclusion, this study suggests that hypoxia-induced expression of maspin might be associated with poor prognosis of OCCC. Nevertheless, this hypothesis should be investigated by further basic research using various cell lines and large-scale clinical studies.

\section{Conflicts of Interest}

All Authors declare no conflicts of interest in relation to this study.

\section{Authors' Contributions}

HSK designed this study. EJL, SJP, and HSK collected the data. EJL, SJP, CL, GWY, JWK and HSK analyzed the results. EJL, GWY, JWK and HSK contributed to manuscript writing. All Authors read and approved the final manuscript.

\section{Acknowledgements}

The Authors sincerely appreciate the assistance provided by Samyang Biopharmaceuticals Corp. in donating paclitaxel for this study.

\section{Supplementary Material}

Available at: https://docs.google.com/document/d/1FAIcBj14_ttV ptMy-qd6i2sRzr0moa9eBbxmq60X0Qc/edit?usp=sharing

\section{References}

1 Romero I and Bast RC Jr: Minireview: human ovarian cancer: biology, current management, and paths to personalizing therapy. Endocrinology 153(4): 1593-1602, 2012. PMID: 22416079. DOI: $10.1210 /$ en.2011-2123

2 Köbel M, Kalloger SE, Boyd N, McKinney S, Mehl E, Palmer C, Leung S, Bowen NJ, Ionescu DN, Rajput A, Prentice LM, Miller D, Santos J, Swenerton K, Gilks CB and Huntsman D: Ovarian carcinoma subtypes are different diseases: implications for biomarker studies. PLoS Med 5(12): e232, 2008. PMID: 19053170. DOI: 10.1371/journal.pmed.0050232

3 Lee YY, Kim TJ, Kim MJ, Kim HJ, Song T, Kim MK, Choi CH, Lee JW, Bae DS and Kim BG: Prognosis of ovarian clear cell 
carcinoma compared to other histological subtypes: a metaanalysis. Gynecol Oncol 122(3): 541-547, 2011. PMID: 21640372. DOI: $10.1016 /$ j.ygyno.2011 05.009

4 Graeber TG, Osmanian C, Jacks T, Housman DE, Koch CJ, Lowe SW and Giaccia AJ: Hypoxia-mediated selection of cells with diminished apoptotic potential in solid tumours. Nature 379(6560): 88-91, 1996. PMID: 8538748. DOI: 10.1038/ $379088 \mathrm{a} 0$

5 De Jaeger K, Kavanagh MC and Hill RP: Relationship of hypoxia to metastatic ability in rodent tumours. Br J Cancer 84(9): 1280-1285, 2001. PMID: 11336482. DOI: 10.1054/bjoc. 2001.1743

6 Fujiwara K, Shintani D and Nishikawa T: Clear-cell carcinoma of the ovary. Ann Oncol 27 Suppl 1: i50-i52, 2016. PMID: 27141072. DOI: 10.1093/annonc/mdw086

7 Mandai M, Yamaguchi K, Matsumura N, Baba T and Konishi I: Ovarian cancer in endometriosis: molecular biology, pathology, and clinical management. Int J Clin Oncol 14(5): 383-391, 2009. PMID: 19856044. DOI: 10.1007/s10147-009-0935-y

8 Piao J, Lee EJ and Lee M: Association between pelvic inflammatory disease and risk of ovarian cancer: An updated meta-analysis. Gynecol Oncol 157(2): 542-548, 2020. PMID: 32037193. DOI: $10.1016 /$ j.ygyno.2020.02.002

9 Komatsu H, Oishi T, Itamochi H, Shimada M, Sato S, Chikumi J, Sato S, Nonaka M, Sawada M, Wakahara M, Umekita Y and Harada T: Serum vascular endothelial growth factor-A as a prognostic biomarker for epithelial ovarian cancer. Int J Gynecol Cancer 27(7): 1325-1332, 2017. PMID: 28557832. DOI: 10.1097/ IGC.0000000000001027

10 Brocato J, Chervona $\mathrm{Y}$ and Costa M: Molecular responses to hypoxia-inducible factor $1 \alpha$ and beyond. Mol Pharmacol 85(5): 651-657, 2014. PMID: 24569087. DOI: 10.1124/mol.113.089623

11 Guo BQ and Lu WQ: The prognostic significance of high/positive expression of tissue VEGF in ovarian cancer. Oncotarget 9(55): 30552-30560, 2018. PMID: 30093968. DOI: 10.18632/oncotarget.25702

12 Lau DH, Lewis AD, Ehsan MN and Sikic BI: Multifactorial mechanisms associated with broad cross-resistance of ovarian carcinoma cells selected by cyanomorpholino doxorubicin. Cancer Res 51(19): 5181-5187, 1991. PMID: 1717140.

13 Livak KJ and Schmittgen TD: Analysis of relative gene expression data using real-time quantitative PCR and the 2(Delta Delta C(T)) Method. Methods 25(4): 402-408, 2001. PMID: 11846609. DOI: 10.1006/meth.2001.1262

14 Lee HJ, Do JH, Bae S, Yang S, Zhang X, Lee A, Choi YJ, Park DC and Ahn WS: Immunohistochemical evidence for the overexpression of Glutathione peroxidase 3 in clear cell type ovarian adenocarcinoma. Med Oncol 28 Suppl 1: S522-S527, 2011. PMID: 20730571. DOI: 10.1007/s12032-010-9659-0

15 Comhair SA and Erzurum SC: The regulation and role of extracellular glutathione peroxidase. Antioxid Redox Signal 7(12): 72-79, 2005. PMID: 15650397. DOI: 10.1089/ars.2005.7.72
16 Zhang M, Volpert O, Shi YH and Bouck N: Maspin is an angiogenesis inhibitor. Nat Med 6(2): 196-199, 2000. PMID: 10655109. DOI: $10.1038 / 72303$

17 Klasa-Mazurkiewicz D, Narkiewicz J, Milczek T, Lipińska B and Emerich J: Maspin overexpression correlates with positive response to primary chemotherapy in ovarian cancer patients. Gynecol Oncol 113(1): 91-98, 2009. PMID: 19193429. DOI: 10.1016/j.ygyno.2008.12.038

18 Solomon LA, Munkarah AR, Schimp VL, Arabi MH, Morris RT, Nassar H and Ali-Fehmi R: Maspin expression and localization impact on angiogenesis and prognosis in ovarian cancer. Gynecol Oncol 101(3): 385-389, 2006. PMID: 16443262. DOI: 10.1016/j.ygyno.2005.11.049

19 Sood AK, Fletcher MS, Gruman LM, Coffin JE, Jabbari S, Khalkhali-Ellis Z, Arbour N, Seftor EA and Hendrix MJ: The paradoxical expression of maspin in ovarian carcinoma. Clin Cancer Res 8(9): 2924-2932, 2002. PMID: 12231537.

20 Surowiak P, Materna V, Drag-Zalesinska M, Wojnar A, Kaplenko I, Spaczyński M, Dietel M, Zabel M and Lage H: Maspin expression is characteristic for cisplatin-sensitive ovarian cancer cells and for ovarian cancer cases of longer survival rates. Int J Gynecol Pathol 25(2): 131-139, 2006. PMID: 16633061. DOI: $10.1097 / 01 . p g p .0000183050 .30212 .2 \mathrm{f}$

21 Abd El-Wahed MM: Expression and subcellular localization of maspin in human ovarian epithelial neoplasms: correlation with clinicopathologic features. J Egypt Natl Canc Inst 17(3): 173183, 2005. PMID: 16799655.

22 Nakashima D, Uzawa K, Kasamatsu A, Koike H, Endo Y, Saito K, Hashitani S, Numata T, Urade M and Tanzawa H: Protein expression profiling identifies maspin and stathmin as potential biomarkers of adenoid cystic carcinoma of the salivary glands. Int J Cancer 118(3): 704-713, 2006. PMID: 16094606. DOI: $10.1002 / \mathrm{ijc} .21318$

23 Carney BK, Caruso Silva V and Cassimeris L: The microtubule cytoskeleton is required for a G2 cell cycle delay in cancer cells lacking stathmin and p53. Cytoskeleton (Hoboken) 69(5): 278289, 2012. PMID: 22407961. DOI: 10.1002/cm.21024

24 Song TF, Zhang ZF, Liu L, Yang T, Jiang J and Li P: Small interfering RNA-mediated silencing of heat shock protein 27 (HSP27) Increases chemosensitivity to paclitaxel by increasing production of reactive oxygen species in human ovarian cancer cells (HO8910). J Int Med Res 37(5): 1375-1388, 2009. PMID: 19930842. DOI: $10.1177 / 147323000903700512$

Received September 7, 2021

Revised October 11, 2021

Accepted October 12, 2021 